\title{
UEBER DIE SUBSTANTIVISCHE ANWENDUNG DER BILDUNGEN MIT -lîh IN DER BEDEUTUNG 'JEDER' BIS ZUM 11. JAHRH.
}

Grimm hat in der d. gr. II, 569 und III, 53 tiber die construction der mit th zusammengesetzten wörter nicht orschöpfend gehandelt, auch gibt er tuber diesen gegenstand nicht durchaus richtiges. Erdmann, Unters. tiber die syntax d. spr. Otfr. II, 154, beschränkt sich darauf, auf Grimm zu verweisen. Die folgende untersuchung stellt sich zur aufgabe, die angegebene construction nach allen seiten hin zu erlăutern.

Da der adjectivische gebrauch sich nicht von dem anderer adjective unterscheidet, so werde ich im folgenden nur die substantivischen bildungen mit tih behandeln.

Das einfache lih in der bedeutung 'jeder' ist nicht denkbar; wo es dennoch vorzukommen scheint, mussen wir uns nach einer andern erklärung umsehen; es wird weiter unten VI. davon die rede sein. Die einfachste vorkommende form ist gelih, allein ich bemerke im voraus, dass diese form nur in verbindung mit einem genetiv vorkommt, während es alleinstehend eine weiterbildung durch den zusatz eo verlangt. Die ubrigen bildungen mit lih stimmen in der construction mit gelih überein, und deshalb sind sie in der nachfolgenden sammlung in gleiche reihe mit gelih gestellt, jedoch sind die citate für das letztere durch einen * ausgezeichnet.

Es ergeben sich nun folgende sieben constructionen von gelih und seinen compositis:

I. ohne abhängigen genetiv;

II. ohne abhängigen genetiv, mit folgendem relativ; 
III. mit abh. gen. eines pronomen;

IV. mit abh. gen. eines substantivum;

V. im plural mit abh. gen. eines plural;

VI. uneigentliche composition mit -lih;

VII. adverbiale bestimmungen des ortes und der zeit, gebildet mit gelih.

\section{Ohne abhängigen genetiv.}

\section{A. masc.:}

Ich lisse zunächst die belege folgen:

a) nom.: ni drôstet iuih in thiu thing, thaz iagelih ist ediling. Otfr. 1, 23, 45 (Kelle); I, 23, 58; I, 24, 3; II, 2, 10 ; II, 3,66 ; II, 8,25 ; II, 8,32 ; II, 9,22 ; II, 23,25 ; III, 5, 8; III, 15, 51; III, 17, 42; III, 17, 44; III, 17, 45; III, 17,47 ; III, 22, 40; IV, 6, 39; IV, 15, 52; IV, 16, 54; V, 4,$11 ; \mathrm{V}, 4,57 ; \mathrm{V}, 6,33 ; \mathrm{V}, 9,53 ; \mathrm{V}, 11,44 ; \mathrm{V}, 11,49$; V, 20, 109; V, 23, 294; Psalm (Heinzel u. Scherer) XI, 3; XXVIII, 9; Symb. apost. (Heinz. u. Scher.) 1; Summa theol. 29, 9; Capell. (Hattem.) $351 \mathrm{~b} ; 370 \mathrm{a}$; Weissenb. cat.; Tat. (Sievers) 119, 13; Notk. cat.; Boeth. (Graff nach seiten) 6; $12 ; 102 ; 103$; Beow. (Grein) $874 ; 987 ; 984 ; 1165$.

b) acc.: ênen gewiliken nd. glaube (Mtillenhoff); Otfr. I, 27, 50; III, 6, 23.

c) dat.: vone diu gibit tiu nutura iogelichemo Boeth. 153; 220; Psalm XXII, 15; XXXVI, 6; CIII, 16.

Die obliquen casus des masc. treten an zahl auffälig gegen den nom. zurtick. Dieser hat bei Otfr. unter 27 fällen 23 mal keine flexionsendung, während sich in den tubrigen schriften das verhältnis umkehrt: flectiert zu unflectiert verhält sich ungefähr wie 10:4. Zu Tatian 119,13 inti uuarlta ioginuelîh in sin hûs et reversi sunt unusquisque in domum suam bemerke ich, dass unusquisque sonst häufig durch das zusammengesetzte einero giuuelih ausgedriickt wird. Mit dem artikcl ein findet sich der nom. nur in der summa, der acc. im nd. gl., der dat. zweimal in den Psalmen. Der gen. kommt alleinstehend gar nicht vor. Es sind noch zwei fälle hinzuzufugen, wo iogelih im plural steht: Capell. 294 b iogeliche durh sih 
sunderigo säzen und Wessobr. gl. u. b. (Denkm. XCV) iegelîchen als dat. Die beschränkte anwendung des plur. erklärt sich daraus, dass schon die form des sing. eine mehrheit in sich schliesst.

Eine eigentümliche eigenschaft der bildungen mit hih ist ihre fähigkeit, einero und allero als verstärkung $\mathrm{zu}$ sich $\mathrm{zu}$ nehmen. Tat. 80,3 thaz iro einero ginuelh ; 99, 5; 176, 3; 95,$5 ; 67,15 ; 90,6$. Dass hier nur eine verstärkung vorliegt - und so erklärt es Graff ahd. spr. II, 109 a und Sievers im glossar zu Tat. - geht klar hervor aus allero giuuelih fon iu Tat. 67, 15. Wenn gelih mit dem zusatz allero 'jeder von allen' bedeutete, so könnte nicht fon iu hinzugefugt werden. Vielleicht ist ubrigens die tatianische verstärkung durch einero bei giuuelih weiter nichts, als eine nachahmung des ags. ânra gehwylc (Grein, Sprachsch. I, 31, Lye s. v. ânra).

\section{B. neutr.:}

a) nom.: $x \alpha \tau \eta \gamma$. (Hatt.) $404 \mathrm{~b}$ uuanda man zeigôn mag uuar iogelîchez liget.

b) dat. Notk. v. d. mus. (Hatt.) $587 \mathrm{~b} d a z$ an iogelichemo (i. e. alphabet) sî diapason.

c) nom. plur.: Tat. 240, 1 oba thiu alliu giscribaniu vvurdir suntringon giuuelichiu.

d) verstärkt durch einero: Tat. 45, 4 thar uuârun steinînu uuezarfaz sehsu gisezitu after subernesse thero Iudeôno thiu bihatên mohtun einero giuuelih zuei mez odo thriu.

In Tat. 240, 1 ist suntringon ginuelichiu die ubersetzung vos per singula, und so der plur. als nachahmung des lat. erklärlich.

\section{C. fem.:}

a) nom.: Capell. 344b iogelichiu dia andara.

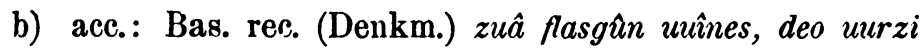
ana zi rîbanne: eogiuuelîhha suntringûn.

Das fem., nur zweimal im nom. und acc. vorkommend, hat die flexion bewahrt. Das erste beispiel iogelichiu dia andara zeigt ein reciprokes verhältnis, wie gr. $\dot{\alpha} \lambda \lambda \dot{\eta} \lambda \omega v$. Das zweite, eoguuelihha suntringân, bedeutet unam quamque per se. 
II. Ohne abhängigen gen., mit folgendem relativ.

a) nom. masc.: thaz iogiuuelîh, ther sih gibilgit zi sînemo bruoder, ther ist sculdig duomes Tat. 26, 2; 28, 1; 42, 1; 87, 4; 118, 3; 135, 15: 143, 3; 171, 3; 194, 7; Psalm CXVIII, U 153; CXVIII, R 135.

b) dat. masc.: iogilichemo, themo Tat. 108,$6 ; 149,8$.

e) neutr.: Tat. 167, 1 iogiuuelihaz, thaz.

d) verstärkt durch allero: allero giuuelihh, thie bitit inph $\hat{a}$ hit Tat. 40, 5; 43, 1; 43, 2; 44, 21; 131, 14; 198, 1; VII Matth. XIII, 7 (Endl. u. Hoffmann 2. aufl.).

Es findet sich in dieser classe nur das masc. und neutr. Der nom. sing. masc. ist zweimal in den Psalmen mit dem unbestimmten artikel versehen. Verstärkung durch allero hat nur im Tat. und einmal im Matth. statt.

III. Mit abhäng. gen. eines pronomen.

Wenn gelih mit dem genetiv eines pronomen oder substantivum verbunden ist, so nimmt es stets das genus des abhängigen gen. an.

A. masc: dero iogelîh habêt sina kenennida Notk. cat.; Boeth. 8; 133; Otfr. IV, 7, 45; V, 25, 65; Tat. 29, 2; 151, 4; Psalm CXI, 5; CXXXVIII, 19; Tat. 103, 4. Es kommen vor die abhängigen genetive dero, iuer, iro, sulichero, und zwar vorgestellt; jedoch bei dem durch einero verstärkten giuuelih Tat. 103, 4 ist iuuar nachgestellt.

B. neutr.: also ouh an den liden sunderingiu geba ist iro iogeliches Psalm XXXII, 15; Boeth. 166; 131; 216. Der abhängige gen. ist stets vorgestellt.

C. fom.: dar iogelichiu iro (i. o. musarum) rarta bechnâta Capell. 285 b; Boeth. 8; Capell. 276 b. Zu bemerken ist das erste beispiel, welches ausnahmsweise einen nachgestellten gen. zeigt.

IV, Mit abhäng. gen. eines substantivum.

\section{A. masc.}

1) a. nom : scal manno gilîh fona deru moliu arstên *Musp. 81; *Rithm. teut. 50; Otfr. II, 8, 47; Allerh. (Denkm. LXX); Hel. 2593; 2733; 3875; 4589; 4597; Beow. 9; 1104; 2887; $266 ; 985 ; 1166 ; 1673 ; 2215 ; 2233 ;$ Otfr. $\mathrm{H}, 19,12$. 
b. acc: iogelichen dero uerlornon Boetl. 26; Hel. 352; 31189 ; Beow. 936; 2250; 2516; 148.

c. dat: unde iogelichemo sinero keloubegen Psalm CI, 1; HIel. $907 ; 1700 ; 1714 ; 1750 ; 1963 ; 2036 ; 2490 ; 4378 ; 4775$; Breow. $3057 ; 1050 ; 2891 ; 412 ; 768 ; 784$ u. ö.

d. gen: Isid. (Holzm.) 43, 22 in isaies buohhum eo chihuueliihhes dero heideo sundric undarscheit; Hel. 2880; 3200; 4116 ; Beow. 2053; 2224; $732 ; 1396$.

e. nom. masc. mit abhäng. gen. sing: that mî̀e mênềôs mankunnies gehwilik Hel. 1505; 4050.

2) verstärkt durch allero.

a. nom: ni allero manno kilih ze demo mahale sculi Musp. 34; Freis. pater nost.; Ezzo 5, 1; exhort. ad pleb. chr.; Hel. 1418; $1537 ; 1754 ; 2050 ; 2065 ; 2616 ; 2618 ; 3216 ; 4250 ; 4377$.

b. dat: pi diu ist durft mihhil allero manno uuelihhemo Musp. 18; Freis. pat. nost.

Auffällig zahlreich bieten sich die beispiele aus Hel. Nur einmal, bei Otfr., ist der nom. flectiert. Der abhängige gen. ist liberwiegend vorgestellt, etwa im verhältnis von $6: 1$. Bemerkenswert sind die beiden stellen aus Hel, wo gehwilkk mit dem gen. sing. verbunden ist, das zweite auch deshalb noch, weil das verbum dem sinne nach construiert im plur. steht. Verstärkung durch allaro findet sich besonders oft im Hel. Auf Grimms falsche erklärung von thegeno gelih, Rithm. tout, weist schon Graff ahd. ahd. spr. II, 112, hin.

\section{B. neutr.}

dara scal queman chunno kilihhaz * Musp. 32; Beow. 2433; * de Heinrico; * Otfr. I, 18, 5; Hel. 5255; Beow. 2608; Hel. 1343; Beow. 2094; Hel.1463; 4155; Beow. 98; 1090. Verstärkt durch allero: Musp. 92; Hel. 975; 1412; 3852; VIII Matth. 17; Hel.1690.

Der gen. ist hier stets vorgestellt. $\mathrm{Zu}$ chunno kilihhaz Musp. 32 bemerke ich folgendes: es ist mir kein beispiel in der altdeutschen literatur vorgekommen, wo der abhängige genetiv von gelih getrennt ist. Die umstellung Denkm. III, dara scal chunno queman io kilihhaz, wo io conjectur ist, ergibt sich also als eine syntactische unmöglichkeit, besonders, da durch die cäsur eine noch stärkere trennung hervorgebracht wird. Dichterische licenz kann ebenfalls nicht zugegeben 
werden, da sich auch im Hel. und Beow. kein analogon dafür findet.

\author{
C. fem.
}

a. acc. daz er rahhôno uuel̂hha rehto arteile Musp. 64 ; 69 ; Hel. 56; Beow. 1705.

b. dat: an dero mâzon iogelîchero sint zuei frontes Capell. $322 \mathrm{~b}$; de vocat. gent.; Beow. 806 .

c. Verstärkt durch allarô: thô lêt hi that werod thanan an alloro halba gehwilika Hel. 1987.

Das fem. tritt äusserst selten in der angegebenen construction auf; der abhäng. gen. ist stets vorgestellt. Das citat aus dem Heliand halte ich für hierhergehörig, da ich nicht den grund einsehe, aus welchem Heyne im gloss. zu Hel. gihwilika als acc. plur. auffassen will.

Ich komme hier nochmals auf die von Sievers angeregte, von Emil Henrici, z. gescb. d. mhd. lyrik s. 63 aufgenommene und im anz. f. d. alt. II, 147 kritisierte frage uber Denkm. VIII zurlick. Einfaches gilih mit abhäng. gen. kommt vor: Musp. 81: scal manno gilih fona deru moltu arstên; Rithm. teut. 50: thâr faht thegeno gelìh nichein sôsô Hludû̀g; Musp. 34: ni allero manno kilih ze demo mahale sculi; Musp. 32: dara scal queman chunno kilihhaz; de Heinr: cui non fecisset Heinrich allero rehto gilih; Otfr. I, 18, 5: Thoh mir megi lidolihh sprechan uuorto gilîh. Fur den acc. fem. habe ich keine belege aufgefunden, wir müssen daher die andern composita bei der fraglichen tubersetzung zu rate ziehen; und da zeigt sich donn acc. fem. -lîhha: rahhôno uuelìhha, thiodô gehwilikka, peóda gehwylce. êrôno gilîh ist also tatsächlich unmöglich, da gelîh stets das genus des abhängigen gen. hat, und da der acc. sing. fem. der pronominalen declination im ahd. durchaus flectirt ist. êrôno gilih als compositum hinzustellen ist auch nicht möglich, da auch bei der composition mit - lih , wo dieselbe bei femininis vorkommt, die flexionsendung des acc. erhalten ist, so gloss. Ker. $160 \mathrm{~b}$ : in fêrônolîhha, $200 \mathrm{a}$ : in fêranolîhha. Die alte sprache scheint liberhaupt vor der verbindung eines fem. mit gelih eine scheu gehabt zu haben; denn erst bei Notk. und in der Bamb. beichte findet sich dieselbe einige male wider (siehe unten), so jedoch, dass die genetivendung verstlummelt und stets die verstärkung allero hinzugefügt ist. Eine ausnahme 
biilden nur einige adverbiale bestimmungen der zeit und des orrtes, z. b. in zîtelîh, in aller stetegelîch, bei welchen das bewrustsein für das ursprüngliche genus leicht verschwinden konnte (siehe unten). Das im anz. f. d. alt. aan. angezogene in zîtelih oder gar ein mhd. citat ist daher nicht geeignet, die riichtigkeit von êrôno gilih zu beweisen. Es kann vielmehr niicht geleugnet werden, dass Haupt einen grammatischen feshler gemacht hat, und dass somit Denkm. VIII nicht in die alhd. literatur gehört. Wenn Steinmeyer etwa noch ein neutrum êrônolih an der fraglichen stelle vorschlagen wollte, so wäre es seine sache, dafür aus dem 8 . jh. belege zu finden; ic; kann solche nicht nachweisen, halte aber das schon aufgefuhrte in fêrônolihka für einen positiven gegenbeweis.

V. Im plural mit abhäng. gen. eines plural.

a. acc. plur. neutr.: $x \alpha \tau$. $411 \mathrm{~b}$ tero sibin quantitatum iogelîcho chît man ebenmichel unde uneben michel.

b. dat. plur. masc.: kuningo gihwil̂kun hêmsitteandiun Hel. $342 ; 1008 ; 1020 ; 1113 ; 1618$. Der plural findet sich also im Hel. ziemlich häufig, selten im hd., wegen der schon oben betonten pluralbedeutung des sing. von gelih. In den $x \alpha \tau$. $411 \mathrm{~b}$ muss iogelîcho als neutr. plur. genommen werden, wenn man nicht etwa einen schreibfehler statt iogehicha annehmen will. Der gen. ist stets vorgestellt.

VI. Uneigentliche composition mit -lih.

Der umstand, dass bei einigen der composita -lîh, bei andern gelih den zweiten bestandteil bildet, gewährt uns einen fingerzeig, wie man sich diese wörter entstanden denken muss. Sievers bemerkt mir: "th in der bedeutung von galih existiert nur in der einbildung älterer lexicographen und grammatiker. lîh heisst nur 'körper', 'äusseres', und galih also 'dasselbe äussere habend'; das $g a$ ist unbedingt notwendig fur das zustandekommen des adjectivbegriffes, (s. Zimmer, Nominalsuff. $a$ und $a \mathrm{~S} .231 \mathrm{f}$.). Auch die scheinbaren composita sind also als gelih mit vorhergehendem genetiv zu betrachten. Bei der verschmelzung der beiden bestandteile ist jedoch schon frihzeitig verstummelung eingetreten: bald ist die vorsilbe gi geschwunden, bald die genetivendung verstammelt, bald auch bei- 
des eingetreten. So begegnen nebeneinander die formen mannogelîh, manniglîh, mannilîh u. 8. w.

A. masc.und neutr:

1. mannolîh.

mannolih, mannilì, manniclìh, mannelih, mannalih, mennisglih, mangelih sind die vorkommenden formen.

a) nom.: mannolih Psalm CIII, 23; Otfi. I, 6, 15; I, 23, $12 ; \pi \varepsilon \rho \grave{\varepsilon} \varrho \mu .495 \mathrm{~b} ; 496 \mathrm{a} ; 519 \mathrm{~b} ; 525 \mathrm{~b} ;$ Boëth. 88;109; 113 ; mannilih: Otfr. prol. ad. Lud. 31 ; I, 3, 40; I, 23, 8; I, 23, 59; II, 2, 9; II, 4, 76; II, 17, 24; II, 18, 15; III, 6, 44; III, 9, 7; III, 21, 23; IV, 13, 9; V, 1, 18; V 1, 24; V, 1, 29; V, 1, 36; $\mathrm{V}, 1,41 ; \mathrm{V}, 1,47 ; \mathrm{V}, 12,19 ; \mathrm{V}, 19,51 ;$ manniclihh: Notk. cat.; Psalm II, 12; L, 19; CXII, 1; CVIII, C, 20; CXVIII, G, 55; CXLIV, 21; mannelih: Notk. cat.; mannalih: Otfi. V, 7, 54; mennisglih: Bamb. gl. u. b.; iro mannolîh: Boeth. 102; aller mennisglih Bamb. gl. u. b.; aller mangelîh Alem. gl. u. b.

b) acc.: mannolîchen Boetb. $38 ; 110 ; 192 ;$ mannilichan: Otfr. II, 12, 16; manniclichen: Psalm CXVIII, E, 39; mannighichon psalm IX, 9.

c) gen.: mannoliches: Boeth. $68 ; 78 ; 269$; manniliches: Otfi. I, 18, 31; III, 20, 39; manniglitches: Psalm XLVIII, 20; manniclàches: Psalm CXLV, 2.

d) dat.: mannolichemo: Boeth. 206; 217; 254; Capell. $340 \mathrm{a}$; manniclichemo: Psalm VII, 12; CXIV, 5; CXVIII, A3; CXXVII, 3; CXXIX, 3; mannelichemo: Capell. 304 b.

Das wort mannolih ist ganz ubergegangen zu der bedeutung von iogelih, wie Boeth. 102 zeigt, wo dem mannolih ein abhängiger gen. iro vorgesetat ist. Otfrid gebraucht uberwiegend die form mannilih, einmal mannalih, sonst auch mannolih; die psalmen haben mannichih, nur einmal mannolih. Ausser der vocalanähnelung $\mathrm{zu} i$ findet sich schwächung zu $e$ bei Notker, einmal die form mangelih Alem. gl. u. b., wo die silbe -no ausgefallen ist. Verstärkung durch allero findet nur im nom. statt. Die form mennisglih Bamb. gl. u. b. ist entstanden aus ursprünglichem menniscôno-gelih, und man hat als zwischenform, analog dem mangelih, ein mennisco-gelih anzunehmen, aus welchem nach abermaligem ausfall des 0 unsere form entstanden ist. 
Was Grimms bemerkung a. a. o. hetrifft, nach weleher die obliquen casus nur selten auftreten, so gibt die zusammenstellung doch ziemlich zahlreiche belege, auch führt Grimm die form manniclich nicht auf. Ich will noch erwähnen Otfr. prol. ad Lud. 9 mannogilih, wo die aneinanderfügung der bestandteile noch kaum eomposition genannt werden kann. Sämmtliche obliquen casus haben ihre flexionsendung bewahrt.

2) dingolîh, dingelîh, tingolîh. $449 \mathrm{a} ; 379 \mathrm{~b}$. $245 ; 252$.

b) acc.: Capell. $340 \mathrm{a}$; Boeth. 105; 152; 213; 219;

c) gen.: Boeth. 148.

d) dat.: $\pi \varepsilon \varrho \grave{\varepsilon} \varepsilon \rho \mu .470 \mathrm{~b} ; 523 \mathrm{a}$.

Verstärkt durch allero:

a) nom.: Boetb. $107 ; 154 ; 210$.

b) acc.: Boeth. $151 ; 264 ;$ xat. 391 a; $403 \mathrm{a}$.

c) gen.: Boeth. $72 ; 81 ; 93 ; 156$.

dingolih kommt nur bei Notker vor, überwiegend im Boeth. Zweimal in den $x a \tau$. ist das $o$ zu $e$ geschwächt. Auch hier sind, entgegen Grimms aufstellung, die casus zahlreich vertreten. Die verstärkung durch allero ist fast ebenso häufig, wie das einfache dingolih. Die flexion der casus ist mit ausnahme des acc. erhalten.

\section{3) guotelîh}

und guatigilich. Das wort findet sich ohne verstärkung bei

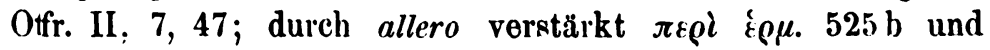
psalm XLIX, 7. Die flexion ist mit ausuahme des acc. erialten.

\section{4) friuntilîh}

begegnet nur bei Otfr. V, 1, 30; V, 1, 35; V, 1, 42; V, 1, 48; $\mathrm{V}, 4,3$, und auch hier nur im nom.

5) rehtolîh.

Notk. v. d. vern. $543 \mathrm{~b}$ allero rehtolih zimig. Hierher kann min auch, falls man es als compositum auffassen will, de Hoinr. cui non fecisset Heinrich allero rehto gilich rechnen. 
Die vorstehende stelle aus Notk. zeigt ubrigens, dass -lih in der bedeutung durchaus mit al synonym ist; es geht dies hervor aus den gleich folgenden worten a. a. 0. $546 \mathrm{a}$ : omne iustum honestum: alliz reht zimilih; omne iustum bonum, allez reht cuot.

6) strîtolîh. - Capell. 339 a verstärkt durch allero.

7) teilelîh. - Boeth. 135 und 256.

8) uuegelîh - Psalm XXXV, 5 im dat.

9) dieticlîh - Psalm CXLVII, 20 im dat.

10) gotelih. - Capell. $370 \mathrm{~b}$ verstärkt durch allero.

11) boumelîh - Boeth. 153; Psalm CIV, 33 boumegihh.

12) criutelih. - Boeth. 153.

13) rosselih - Psalm XXXI, 9.

14) wêwigelîh. - Himm. u. höll. 156 verstärkt durch aller.

15) sangolîh. - Notk. v. d. mus. 587 b.

16) leidogilsh. - Otfr. V, 23, 218.

17) tierlîh. - Boeth. 206 verstärkt durch allero.

18) lidolih. - Otfi. I, 18, 15.

19) ubelolîh. - Boeth. 226 verstärkt durch allero.

Von den gegebenen stellen sind:

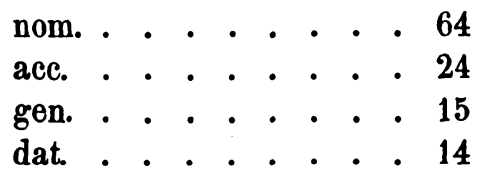

Die obliquen casus zusammen kommen an zahl also dem nom. annähernd gleich. Verstärkung durch allero findet im verhältnis von 1:5 statt; mannolih hat nie ein allero bei sich.

B. fem.:

1) sâldolîh.

Boeth. 231 und 229, beide male durch allero verstärkt. Der erste der angeführten belege bietet eine schwierigkeit. Dass Notk. sâldolih gemäss seiner zusammensetzung als fem. behandelt, geht aus dem zweiten citat hervor: allero sâldolih vuunesâmiu. Boeth. 231 zeigt nun die widergabe eines lateinischen accusativus cum infinitivo, wir sollten also als subject in demselben allero sâldolihha erwarten. Dass Notker trotzdem sâldolih schreibt, findet nur darin seine erklärung, dass 
der acc. c. inf. keine geläufige deutsche construction ist; das vorliegende sâldolîh ist nom., die ganze construction ein nominativus cum infinitivo.

2) skandigelîh.

Himm. u. höll. 152 verstärkt durch aller.

3) egisilîh.

Himm. u. höll. 172 verstärkt durch aller.

4) uuîbilîh.

Otfr. IV, 26, 35 thaz uuîbilîh fon iru quît.

Das fem., nur selten vorkommend, zeigt meist die verstärkung durch allero. Ich habe hier noch eine ganz vereinzelt dastehende anwendung zu erwähuen Boeth. 256 unde daz knôtesta uueiz si, teilelîchen neuueiz si; und ebenda: nioman ne ist sô gehuhtig, daz er alles teilelîches sô uuola gehuge, sô des knôtesten. teilelih bezeichnet hier, wie aus dem zusammenhang hervorgeht, 'singula'. Notker gebraucht es also wie ein substantivum und vergisst bei der widergabe von 'omnium singulorum', dass der begriff' 'omnium' schon in teilelih enthalten ist.

VII. Adverbiale bestimmungen des ortes und der zeit gebildet mit -lih.

Sie gehören der form nach zu den compositis; es empfiehlt sich jedoch, sie allein zu behandeln, da sie bis zum aufgeben der Hlexion formelhaft geworden sind.

1) 'tiberall hin'

ist ausgedrlickt durch in allero endeglih Psalm CIV, 31; im Bamb. gl. u. b. in aller stetegelich.

2) 'nach allen seiten'

wird ausgedritickt durch in fêrônolihh gloss. Ker. $160 \mathrm{~b}, 200 \mathrm{a}$ (Hattem.).

\section{3) 'alle tage'}

wird ausgedruckt: a) durch den genetiv von gelih mit vorgestelltem abhängigen genetiv dago Weissenb. cat.; Hel. 954; $1593 ; 1609 ; 1672 ; 2284 ; 3337 ; 3629 ;$ b) durch den gen. von gelih nit abhängigem gen. dago, verstärkt durch allero. Diese 
verbindung findet sich nur im Hel. 1217; 1254; 1916; 2168; $2346 ; 3333$; 3499; 3781; c) dago und gelı̂ches sind $\mathrm{zu}$ éinom worte verbunden, wobei die ubliche verstimmelung zu tagolihhes, dagolihes, tagelihes stattfindet. Diese ausdrucksweise kommt nur bei Notker vor: Boeth. 43; 49; 77; 109; Capell. 275 a ; 301 b; Psalm VI, 8; VII, 12; XXIV, 11; XLI, 4; XLI, 11; CXXXV, 11; CXLIV, 2. Graff, ahd. sprs. sagt: 'tagalîh steht nur adjectivisch mit folgendem substantivum', ebenso Grimm a. a. o. Diese bemerkung ist, wie das vorkommen der adverbialen genetive zeigt, nicht zutreffend. Aus dem substantivum tagalih hat sich erst das adjectivum tagalihher gebildet, wie es z. b. in der oratio dominica vorkommt.

\section{4) 'für alle zeit'}

wird ausgedrückt: a) durch in mit compositum: in zîtegelihh,

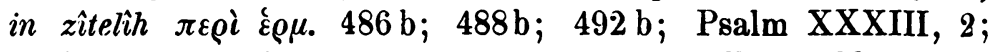
b) im ags. durch instrumentalis mit vorgestelltem abhängigen genetiv: mala gehwylce Beow. 2057.

5) 'in jedem jahre'

wird ausgedrückt durch den gen. von gelich mit vorgestelltem abhängigen gen. jaro geliches Capell. 310 a; Hel. 3812.

6) 'an jedem morgen'

wird ausgedrijckt: a) durch genetiv von gelih mit vorgestellten abhäugigen gen. morgno gehwilikes Hel. 601; b) durch instrtmentalis von gelih mit vorgestelltem abiängigen gen.: morra gehwylce Beow. 2450.

\section{7) 'in jeder nacht'}

wird ausgedrïckt durch nahtegeliches Psalm VI, 7. Man solle erwarten nahtegelicher; die form nahtegelîches ist wol nach analogic von tageliches gebildet, beweist aber inmerhin, we leicht bei den adverbialen bestimmungen, aber nur bei dieseı, das ursprungliche genus des hauptbestandteils vergessen wurds.

BERLIN.

ERNST HENRICI. 